\title{
The features of contrast enhanced ultrasound and BRAF V600E in papillary thyroid carcinoma
}

\author{
Zi-Mei Lin ${ }^{1}$, Cao-Xin Yan ${ }^{1}$, Yue Song ${ }^{1}$, Yu-Rong Hong ${ }^{1}$, Qing Wen ${ }^{1}$, Yong-Yuan Xu ${ }^{1}$, Min-Qiang Pan ${ }^{1}$, \\ Qin $\mathrm{Ye}^{2}$, Pin-Tong Huang ${ }^{1}$ \\ ${ }^{1}$ Department of Ultrasound in Medicine, ${ }^{2}$ Department of Pathology, The Second Affiliated Hospital Zhejiang University School of Medicine, \\ Hangzhou 310009, China \\ Contributions: (I) Conception and design: PT Huang, YR Hong, ZM Lin; (II) Administrative support: PT Huang, YR Hong, ZM Lin; (III) Provision \\ of study materials or patients: Q Ye, MQ Pan, YY Xu, Q Wen, CX Yan, ZM Lin; (IV) Collection and assembly of data: MQ Pan, YY Xu, Q Wen, \\ CX Yan, ZM Lin, YR Hong; (V) Data analysis and interpretation: ZM Lin, Y Song; (VI) Manuscript writing: All authors; (VII) Final approval of \\ manuscript: All authors. \\ Correspondence to: Pin-Tong Huang; Yu-Rong Hong. Department of Ultrasound in Medicine, The Second Affiliated Hospital Zhejiang University \\ School of Medicine, No. 88, Jiefang Road, Hangzhou 310009, China. Email: huangpintong@zju.edu.cn; hyrxwly@163.com.
}

Background: BRAF V600E mutation was proved to be associated with thyroid cancer. Papillary thyroid carcinoma (PTC) with positive BRAF mutation might have a more aggressive behavior. We investigated the correlation of the contrast-enhanced ultrasound (CEUS) features with BRAF 600VE in PTC.

Methods: The medical records of 1,199 patients with 1,315 nodules who underwent CEUS prior to fine needle aspiration (FNA) from January 2016 to March 2018 were retrospectively reviewed. The features of their enhancement were assessed from eight aspects: degree of enhancement, method of enhancement, homogeneity of enhancement, completeness of enhancement, boundary of the enhanced lesions, shape of the enhanced lesions, size of the enhanced lesions, and wash out period of the enhanced lesions. The patients then examined for the BRAF V600E mutation using specimens obtained from FNA.

Results: BRAF mutations were found in 888 of 1,315 nodules. The CEUS features were significantly different between BRAF-positive and BRAF-negative nodules. The BRAF mutation positive nodules were more often with larger size, hypo-enhancement, centripetal enhancement, inhomogeneous enhancement, complete enhancement, blurred boundary, irregular shape, and with wash out period at preoperative CEUS than those without BRAF mutations $(\mathrm{P}<0.001)$. However, no significant correlation was showed in Spearman's rank correlation between the CEUS features and BRAF mutation, except for degree of enhancement, method pattern of enhancement, and completeness of complete enhancement. Multivariate analysis showed that centripetal (OR: 1.465, 95\% CI: 1.129-1.903) and no significant enhancement (OR: 0.790, 95\% CI: 0.639-0.977) were predictive for the presence of BRAF mutations. The sensitivity, specificity, positive predictive value (PPV) and negative predictive value (NPV) of no significant enhancement and centripetal enhancement of CEUS for predicting BRAF mutation were $68.3 \%, 40.0 \%, 91.6 \%, 11.7 \%$, and $72.4 \%, 35.1 \%, 37.8 \%, 70.0 \%$, respectively.

Conclusions: Our study indicated that preoperative thyroid nodule characteristics on CEUS might serve as a useful tool to BRAF mutation in PTC.

Keywords: Thyroid cancer; contrast-enhanced ultrasonography; ultrasound; BRAF mutation

Submitted Sep 10, 2019. Accepted for publication Nov 26, 2019.

doi: $10.21037 /$ jtd.2019.11.78

View this article at: http://dx.doi.org/10.21037/jtd.2019.11.78 


\section{Introduction}

Papillary thyroid carcinoma (PTC) is the most common type of thyroid cancer and accounts for approximately $80-85 \%$ of malignant neoplasms of the thyroid gland $(1,2)$. There are many factors could underlie the prognosis of PTC including patient age, gender, tumor size, histological findings, extrathyroidal extension (ETE), clinical lymph node metastasis (LNM) and remote metastasis (3). BRAF V600E mutation was proved to be associated with thyroid cancer through up regulation of cell division and proliferation leading to tumor genesis $(4,5)$. Furthermore, it was suggested that PTC harboring an activated BRAF mutation might have a more aggressive behavior like ETE, LNM, distant metastasis, advanced TNM stages, and cancer recurrence $(6,7)$. Previous studies have established a statistically significant association between the BRAF mutation and tumor size, advanced clinical stage, multifocality, LNM, ETE, and recurrence in PTC (8-10).

Fine-needle aspiration (FNA) is a first line diagnostic method for thyroid nodules. For the mutation test, cytologic samples from FNA are commonly used. The remaining materials from the needle after FNA are proved to be sufficient cellular material for the BRAF mutation test (11). However, some cytologic results such as non-diagnostic, indeterminate, false-negative, and false-positive results confuse the clinicians (12). Contrast-enhanced ultrasound (CEUS) is adjunct ultrasound (US) imaging technique that is used to differentiate benign from malignant thyroid nodules. Recently, some prospective studies have confirmed the high predictive value of CEUS in identifying malignancy (13-15). According to these results, we aimed to systematically review the relation between the BRAF mutation and the characteristics on CEUS. To the best of our knowledge, no design in this topic was reported.

\section{Methods}

\section{Patients}

This study was approved by the institutional review board at the Second Affiliated Hospital Zhejiang University School of Medicine and waived the need for informed consent based on the retrospective design. We reviewed the medical records of 1,315 cytopathologically-proven, conventional PTC patients who had undergone fine needle aspiration (FNA) from January 2016 to March 2018.

\section{US, CEUS and FNA}

US and CEUS examination were performed by radiologists with 5-20 years of experience in thyroid ultrasound, using a 3-9 MHz transducer (Esaote MyLab 90, Esaote Group, Genova, Italy) equipped with a contrast-specific, continuous-mode software for CEUS. Sulphur hexafluoride (SonoVue ${ }^{\circledR}$, Bracco International, Milan, Italy), was used as ultrasound contrast agent in this study. Every CEUS record of thyroid nodule persists $3 \mathrm{~min}$. The contrast enhancement patterns of the lesions were assessed from the following eight aspects: (I) size of the enhanced lesions: larger and similar; (II) degree of enhancement: nonenhancement, hypo-enhancement, iso-enhancement, and hyper-enhancement.(no significant enhancement : enhancement was lower or similar to the surrounding gland; hyper-enhancement: enhancement was greater than the surrounding gland); (III) patterns of enhancement: centripetal enhancement, centrifuge enhancement and diffused enhancement; (IV) homogeneity of enhancement: inhomogeneous enhancement and homogeneous enhancement; (V) completeness of enhancement: incomplete enhancement and complete enhancement; the so called complete enhancement refers that the entire lesion were filled with contrast medium, while incomplete enhancement refers that the lesion appear region without contrast agent filling in; (VI) wash out period: with wash out period and non-wash out period; (VII) boundary of the enhanced lesions: blurred and well defined; (VIII) shape of the enhanced lesions: irregular and regular.

Ultrasound guided FNAs were performed by the same radiologists with at least 5 years of experience in thyroid nodule FNA. Each nodule underwent FNA without suction with the freehand technique. At least four needle passes were required in order to obtain adequate specimens. The number of needle passes depended on the quality of slides assessed by pathologist immediately. Samples obtained with 23 -gauge needles were expelled on glass slides, smeared, and placed immediately in $95 \%$ ethyl alcohol for Papanicolaou staining. The remaining materials were rinsed in saline for cell-block processing. Samples obtained from the other passages were assessed for molecular testing.

\section{Pathological examination and BRAF mutation analysis}

The FNA specimens were microscopically examined by an experienced pathologist. To distinguish bilaterality from 
multiplicity, we defined multiplicity as more than two lesions of conventional PTC in one lobe, regardless of the presence of bilaterality.

BRAF mutation analysis was performed at the Molecular Diagnostics Laboratory of the Second Affiliated Hospital Zhejiang University School of Medicine. DNA samples for molecular analysis were extracted from FNA specimens. Based on the BRAF sequence, the PCR primers were designed, including forward primer (5'-TCATAATGCTTGCTCTGATAGGA-3') and reverse primer (5'-GGCCAAAAATTTAATCAGTGGA-3'). The detection kits were provided by Amoy Diagnostics Co., Ltd. The sequencing of PCR products was performed using a LightCycler 480 II (Roche Diagnostics, Germany) after DNA extraction and PCR amplification. The Cycle threshold $(\mathrm{Ct})$ value was also obtained. The $\mathrm{Ct}$ value of sample less than 28 was defined as positive, while equal to or greater than 28 was negative. And the amplification curve was also assessed to estimate the amplification signal.

\section{Statistical analysis}

The SPSS for Windows version 13.0 software package (SPSS Inc., Chicago, IL) was used for statistical data analysis. Continuous data are presented as mean (standard deviation) and compared by using Student's $t$ test. Categorical data are presented as percentages and compared using the Chi-square test and the Pearson chi-squared test. The rankings of valuable indicators were compared according to the odds ratios (ORs). All risk ultrasound features that proved to be statistically significant on Chisquare test were analyzed to assess independent association with BRAF mutations using multivariate logistic regression.

\section{Results}

The mean age of the 1,199 PTC patients (316 males and 883 females) was $44.8 \pm 12.4$ years (range, $18-77$ years), and the mean tumor size was $0.83 \pm 0.51 \mathrm{~cm}$ (range, $0.2-4.5 \mathrm{~cm}$ ). Papillary thyroid microcarcinoma (PTMC) measuring $<1 \mathrm{~cm}$ in size was observed in 1,008 cases $(76.7 \%)$. Four patients $(0.3 \%)$ had multiple tumors on one lobe, whereas 116 patients (8.8\%) had bilateral conventional PTC (Table 1).

BRAF V600E mutation was found in 888 of 1,315 nodules $(67.5 \%)$. The difference of contrast enhancement patterns between BRAF-positive and BRAF-negative nodules was shown in Table 2. The differences of contrastenhancement patterns between BRAF-positive and BRAF- negative nodules in eight aspects were all statistically significant $(\mathrm{P}<0.001)$. Most contrast-enhancement patterns of PTCs with positive BRAF mutation were irregular, blurred, no significant enhancement, inhomogeneous enhancement, complete enhancement and with larger size (Figure 1).

However, no significant correlation was showed in Spearman's rank correlation between the CEUS features and BRAF mutation, except for degree of enhancement, method of enhancement, and completeness of enhancement (Table 2). Multivariate analysis showed that centripetal (OR: 1.465 , 95\% CI: $1.129-1.903$ ) and no significant enhancement (OR: 0.790, 95\% CI: 0.639-0.977) were predictive for the presence of BRAF mutations, while the size, homogeneity, completeness, wash-out, boundary, and shape showed no significant differences between positive BRAF mutation PTCs and negative BRAF mutation PTCs (Table 3). The sensitivity, specificity, positive predictive value (PPV) and negative predictive value (NPV) of hypoenhancement and centripetal enhancement for predicting BRAF mutation were $68.3 \%, 40.0 \%, 91.6 \%, 11.7 \%$, and $72.4 \%, 35.1 \%, 37.8 \%, 70.0 \%$, respectively.

\section{Discussion}

PTC is the most common cancer of thyroid and has an overall favorable prognosis with an average 10 years survival rate of $93 \%$ (8). Despite of the excellent prognosis for most patients with PTC, a small percentage of patients experience a more aggressive course of disease (16-18). The BRAF V600E mutation composed of a T to A transversion, which is found in up to $73.4 \%$ of PTCs (19) and results in the substitution of valine for glutamate at position 600 . The V600E mutant BRAF constitutively activates the MAPK pathway to stimulate the tumorigenesis. The BRAF V600E has emerged as a promising diagnostic as well as prognostic indicator of PTC since its initial discovery. Several studies have shown that BRAF V600E mutation is an indicator of poor disease prognosis because it's association with advanced disease stage and cancer recurrence (19-21). It has also been reported that the BRAF V600E mutation could increase not only the aggressive nature of tumor but also the recurrence and mortality rates of PTC (22). Therefore, preoperative determination of BRAF V600E mutation was thought to be useful in determination of the management and operative strategy.

US has been proved to be vital in diagnosing thyroid disease (12-14,23). Previous studies have also shown 
Table 1 Clinicopathologic factors related to BRAF mutation in 1,199 patients with PTC

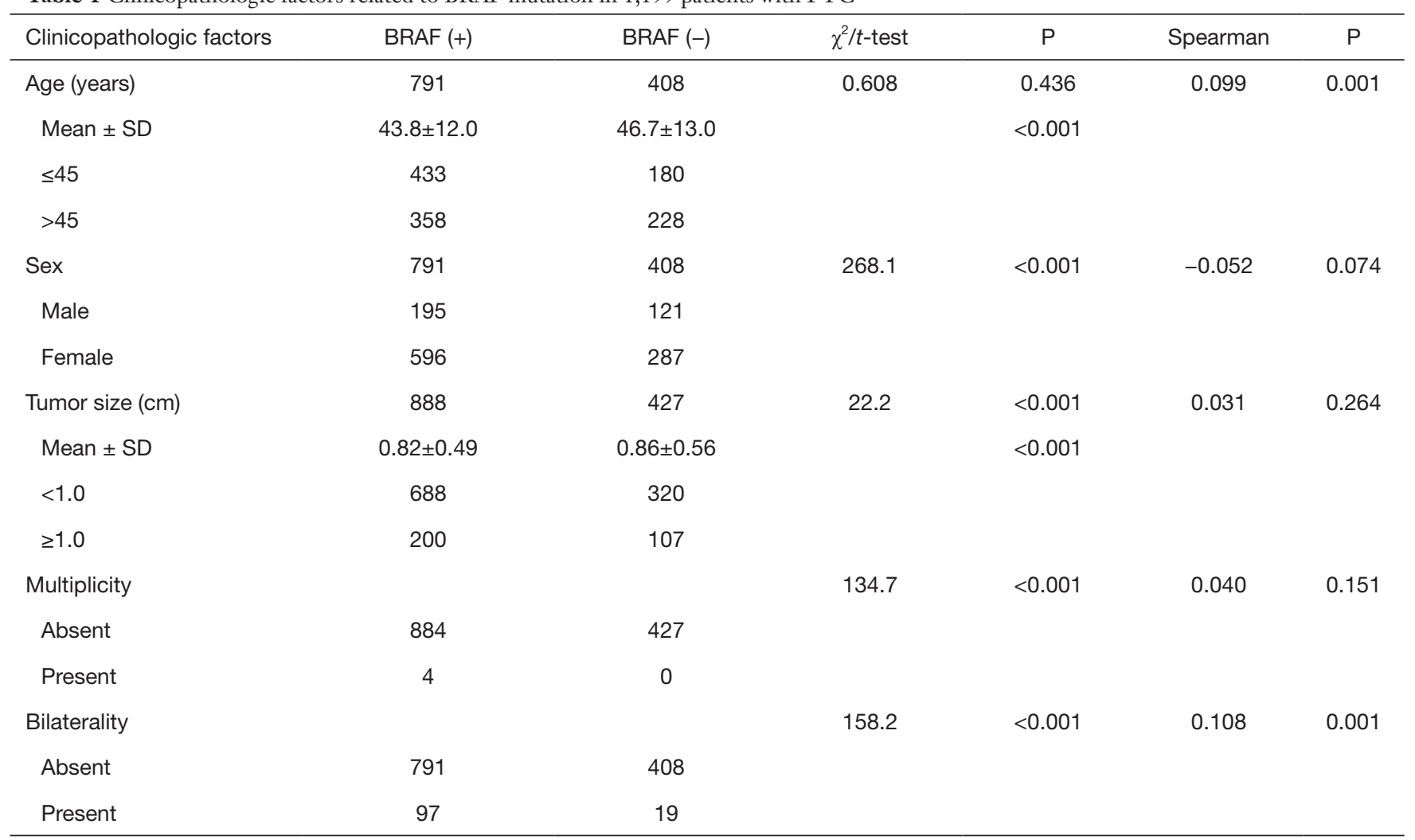

PTC, papillary thyroid carcinoma.

the correlations between the aggressive clinical factors underlying the BRAF mutation and PTC. It was reported that extra-thyroidal extension, multifocality, lymph node metastasis, and advanced TNM were associated with the BRAF V600E mutation in PTC (24). These results suggested the researchers to work on accurate ways to diagnose PTC including the BRAF mutational analysis. Therefore, there is a great need for more accurate preoperative risk stratification systems to inform the BRAF mutation of patients with PTC.

This study examined whether BRAF V600E was associated with CEUS features in PTC. BRAF V600E is only observed in anaplastic carcinoma such as PTC, and not in other thyroid cancers, including follicular carcinoma $(8,9)$. In this study, we only focused on conventional PTC. Most PTCs with BRAF mutation in our study were more often with larger size, no significant enhancement, centripetal, homogeneous, completely enhanced, blurred boundary, irregular shape, and with wash out period at preoperative CEUS than those without BRAF mutations $(\mathrm{P}<0.001)$. However, only centripetal, completely enhanced, and irregular shape were showed significant correlation in Spearman's rank correlation between the CEUS features and BRAF mutation. The pathological sections of malignant nodules revealed the number of sinusoids was much more than that in benign lesions (25). BRAF was proved to play a key role in cell proliferation, differentiation, and apoptosis. Thus, BRAF mutation positive may influence the number of tumor vascular bed, which displayed as centripetal enhancement. Several studies have found that enhancement patterns of thyroid nodules were closely related to lesion size. The nodules smaller than $1 \mathrm{~cm}$ presented no significant and complete enhancement $(5,26)$. In this study, 1,008 (76.6\%) PTCs were smaller than $1 \mathrm{~cm}$. Additionally, the tumors with BRAF mutation invaded outward easily, which might induce the irregular shape.

We also performed a multivariate analysis and revealed that centripetal (OR: 1.465, 95\% CI: 1.129-1.903) and no significant enhancement (OR: 0.790, 95\% CI: 0.639-0.977) were predictors for the presence of BRAF mutations. The vascular bed of PTC is immature when the tumor is small. The pressure of blood vessels increases due to 

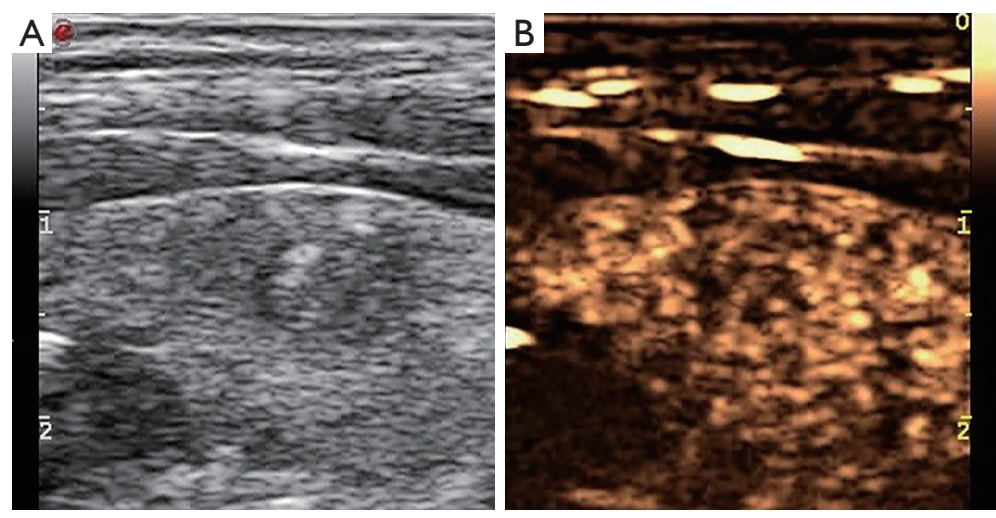

Figure 1 US of a thyroid nodule in a 38-year-old male who underwent fine-needle aspiration. Longitudinal US images demonstrating a hypoechoic nodule in the left thyroid gland (A). The nodule was hypoenhanced on CEUS (B). The cytology was papillary carcinoma and BRAF V600E (+). CEUS, contrast-enhanced ultrasound.

Table 2 The difference of contrast enhancement patterns between BRAF-positive and BRAF-negative nodules

\begin{tabular}{|c|c|c|c|c|c|c|}
\hline CEUS features & BRAF $(+)$ & $\operatorname{BRAF}(-)$ & $\chi^{2}$ & $P$ & Spearman & $P$ \\
\hline Larger & 391 & 180 & & & & \\
\hline Similar & 497 & 247 & & & & \\
\hline Degree of enhancement & & & $1,556.7$ & $<0.001$ & 0.042 & 0.131 \\
\hline Method of enhancement & & & 113.8 & $<0.001$ & 0.077 & 0.005 \\
\hline Centripetal & 336 & 128 & & & & \\
\hline Nonconcentric & 552 & 299 & & & & \\
\hline Completeness of enhancement & & & $1,115.2$ & $<0.001$ & 0.087 & 0.002 \\
\hline Incomplete & 25 & 27 & & & & \\
\hline Complete & 863 & 400 & & & & \\
\hline Wash out period & & & 673.3 & $<0.001$ & -0.010 & 0.730 \\
\hline With wash out period & 129 & 58 & & & & \\
\hline Non-wash out period & 759 & 369 & & & & \\
\hline Boundary of the enhanced lesions & & & 595.6 & $<0.001$ & 0.032 & 0.248 \\
\hline Blurred & 750 & 350 & & & & \\
\hline
\end{tabular}


Table 3 Multivariate analysis of CEUS features with BRAF mutation in PTC

\begin{tabular}{|c|c|c|c|c|}
\hline CEUS features & $\operatorname{BRAF}(+)$ & $\operatorname{BRAF}(-)$ & OR $(95 \% \mathrm{Cl})$ & $\mathrm{P}$ \\
\hline Degree of enhancement (no significant enhancement) & 813 & 377 & $0.790(0.639-0.977)$ & 0.030 \\
\hline Method of enhancement (centripetal) & 336 & 128 & $1.465(1.129-1.903)$ & 0.004 \\
\hline Homogeneity of enhancement (inhomogeneous) & 364 & 164 & $0.806(0.617-1.053)$ & 0.113 \\
\hline Wash out period (with wash out period) & 129 & 58 & $0.814(0.576-1.149)$ & 0.242 \\
\hline Boundary of the enhanced lesions (blurred) & 750 & 350 & $1.030(0.702-1.510)$ & 0.880 \\
\hline Shape of the enhanced lesions (irregular) & 851 & 394 & $1.073(0.398-2.897)$ & 0.889 \\
\hline
\end{tabular}

CEUS, contrast-enhanced ultrasound; PTC, papillary thyroid carcinoma.

the compression of proliferating tumor cells, which also hinders the blood supply in tumors. With the increase of cancer volume, a large number of angiogenesis is induced by angiogenesis factors. Therefore, PTMC is characterized by poor blood supply and uneven distribution of cancer cells results in uneven enhancement (27-29). In this study the pattern of no significant enhancement showed well PPV (91.6\%) in predication of BRAF mutation. Given the prognostic impact of BRAF mutation, these results suggest that PTCs with centripetal and no significant enhancement patterns of CEUS seem to be associated with BRAF mutation, and also with a worse prognosis.

However, there are truly some limitations in this study. First, it's a retrospective analysis. A bias toward larger tumors might exist, because these would be more readily available for collection and genetic analysis. This limitation also allows for a selection bias toward patients with better-documented disease. A prospective welldesigned study was need for the more accurate assessment of the relation between the CEUS features and BRAF mutations. Additionally, this study is based on FNA results. FNA findings were interpreted as non-diagnostic in approximately $10 \%$ to $30 \%$ of FNA results $(30-32)$. Our previous study showed that the sensitivity and specificity of BRAF V600E from FNA was $80 \%$ and $100 \%$ (33). Further study with pathology results would be essential in the future. Thirdly, BRAF mutation was proved to be associated with PTC. However, conventional papillary carcinoma and follicular papillary carcinoma are different disease in the clinical aspect and cytological structure. Thus, the rule of BRAF mutation for differentiation of malignancy according to pathological type should be studied someday.
In summary, our study indicates that CEUS features correlate with BRAF mutation, and CEUS might serve as a useful tool to BRAF mutation in PTC.

\section{Acknowledgments}

Funding: This research was supported by the National Natural Science Foundation of China (Grant No. 81527803, 81420108018), National Key R\&D Program of China (No. 2018YFC0115900), and Zhejiang Science and Technology Project (No. 2019C03077).

\section{Footnote}

Conflicts of Interest: The authors have no conflicts of interest to declare.

Ethical Statement: The authors are accountable for all aspects of the work in ensuring that questions related to the accuracy or integrity of any part of the work are appropriately investigated and resolved. This study was approved by the institutional review board at the Second Affiliated Hospital Zhejiang University School of Medicine and waived the need for informed consent based on the retrospective design.

\section{References}

1. Gharib H, Papini E, Paschke R, et al. American Association of Clinical Endocrinologists, Associazione Medici Endocrinologi, and European Thyroid Association medical guidelines for clinical practice for the diagnosis and management of thyroid nodules: executive summary of 
recommendations. J Endocrinol Invest 2010;33:51-6.

2. Ali SZ. Thyroid cytopathology: Bethesda and beyond. Acta Cytol 2011;55:4-12.

3. Ito Y, Miyauchi A, Kobayashi K, et al. Static and dynamic prognostic factors of papillary thyroid carcinoma. Endocr J 2014;61:1145-51.

4. Kim SW, Lee JI, Kim JW, et al. BRAFV600E mutation analysis in fine-needle aspiration cytology specimens for evaluation of thyroid nodule: a large series in a BRAF V600E-prevalent population. J Clin Endocrinol Metab 2010;95:3693-700.

5. Bentz BG, Miller BT, Holden JA, et al. BRAF V600E mutational analysis of fine needle aspirates correlates with diagnosis of thyroid nodules. Otolaryngol Head Neck Surg 2009;140:709-14.

6. Jemal A, Siegel R, Xu J, et al. Cancer Statistics. CA Cancer J Clin 2010;60:277-300.

7. Kim SJ, Le KE, Myong JP, et al. BRAFV600E mutation is asociated with tumor agresiveness in papillary thyroid cancer. World J Surg 2012;36:310-7.

8. Tufano RP, Teixeira GV, Bishop J, et al. BRAF mutation in papilary thyroid cancer and its value in tailoring initial treatment: asystematic review and meta-analysis. Medicine 2012;91:274-86.

9. Kim TH, Park YJ, Lim JA, et al. The association of the BRAF (V600E) mutation with prognostic factors and poor clinical outcome in papillary thyroid cancer: a metaanalysis. Cancer 2012;118:1764-73.

10. Li C, Lee KC, Schneider EB, et al. BRAFV600E mutation and its association with clinicopathological features of papillary thyroid cancer: a meta-analysis. J Clin Endocrinol Metab 2012;97:4569-70.

11. Di Benedetto G, Fabozzi A, Rinaldi CR. BRAF test and cytological diagnosis with a single fine needle cytology sample. Acta Cytol 2013;57:337-40.

12. Moon HJ, Son E, Kim EK, et al. The diagnostic values of ultrasound and ultrasound-guided fine needle aspiration in subcentimeter-sized thyroid nodules. Ann Surg Oncol 2012;19:52-9.

13. Diao X, Zhan J, Chen L, et al. Quantification of solid hypo-echoic thyroid nodule enhancement with contrastenhanced ultrasound. Transl Cancer Res 2017;6:1078-87.

14. Sui X, Liu HJ, Jia HL, et al. Contrast-enhanced ultrasound and real-time elastography in the differential diagnosis of malignant and benign thyroid nodules. Exp Ther Med 2016;12:783-91.

15. Yan F, Song Z, Du M, et al. Ultrasound molecular imaging for differentiation of benign and malignant tumors in patients. Quant Imaging Med Surg 2018;8:1078-83.

16. Ito $Y$, Higashiyama T, Takamura $Y$, et al. Prognosis of Patients with Papillary Thyroid Carcinoma Showing Postoperative Recurrence to the Central Neck. World J Surg 2011;35:767-72.

17. Huang IC, Chou FF, Liu RT, et al. Long-term outcomes of distant metastasis from differentiated thyroid carcinoma. Clin Endocrinol (Oxf) 2012;76:439-47.

18. Nam SY, Shin JH, Han BK, et al. Preoperative ultrasonographic features of papillary thyroid carcinoma predict biological behavior. J Clin Endocrinol Metab 2013;98:1476-82.

19. Namba H, Nakashima M, Hayashi T, et al. Clinical implication of hot spot BRAF mutation, V599E, in papillary thyroid cancers. J Clin Endocrinol Metab 2003;88:4393-7.

20. Lee JH, Lee ES, Kim YS. Clinicopathologic significance of BRAF V600E mutation in papillary carcinomas of the thyroid: a meta-analysis. Cancer 2007;110:38-46.

21. Xing M. BRAF mutation in thyroid cancer. Endocr Relat Cancer 2005;12:245-62.

22. Xing M, Alzahrani AS, Carson KA, et al. Association between BRAF V600E mutation and mortality in patients with papillary thyroid cancer. JAMA 2013:309:1493-501.

23. Tam AA, Kaya C, Üçler R, et al. Correlation of normal thyroid ultrasonography with thyroid tests. Quant Imaging Med Surg 2015;5:569-74.

24. Liu X, Yan K, Lin X, et al. The association between BRAF (V600E) mutation and pathological features in PTC. Eur Arch Otorhinolaryngol 2014;271:3041-52.

25. Yuan Z, Quan J, Yunxiao Z, et al. Contrast-enhanced ultrasound in the diagnosis of solitary thyroid nodules. J Cancer Res Ther 2015;11:41-5.

26. Gharib H, Papini E, Paschke R, et al. American Association of Clinical Endocrinologists, Associazione Medici Endocrinologi, and European Thyroid Association medical guidelines for clinical practice for the diagnosis and management of thyroid nodules: executive summary of recommendations. J Endocrinol Invest 2010;33:51-6.

27. Jain RK. Normalizing tumor vasculature with antiangiogenic therapy: A new paradigm for combination therapy. Nat Med 2001;7:987-9.

28. Han Z, Lei Z, Li M, et al. Differential diagnosis value of the ultrasound gray scale ratio for papillary thyroid microcarcinomas and micronodular goiters. Quant Imaging Med Surg 2018;8:507-13.

29. Yang C, Qian CW, Zhu HN, et al. Quantitative assessment of blood perfusion in thyroid nodules by 
contrast-enhanced ultrasonography. Chinese Journal of Ultrasonography 2011;20:38-40.

30. Bartolotta TV, Midiri M, Galia M, et al. Qualitative and quantitative evaluation of solitary thyroid nodules with contrast-enhanced ultrasound: initial results. Eur Radiol 2006;16:2234-41.

31. Nemec U, Nemec SF, Novotny C, et al. Quantitative evaluation of contrast-enhanced ultrasound after intravenous administration of a microbubble contrast agent for differentiation of benign and malignant thyroid

Cite this article as: Lin ZM, Yan CX, Song Y, Hong YR, Wen Q, Xu YY, Pan MQ, Ye Q, Huang PT. The features of contrast enhanced ultrasound and BRAF V600E in papillary thyroid carcinoma. J Thorac Dis 2019;11(12):5071-5078. doi: 10.21037/ jtd.2019.11.78 nodules: assessment of diagnostic accuracy. Eur Radiol

2012;22:1357-65.

32. Williams BA, Bullock MJ, Trites JR, et al. Rates of thyroid malignancy by FNA diagnostic category. J Otolaryngol Head Neck Surg 2013;42:61.

33. Hong Y, Luo Z, Wen Q, et al. Use of BRAF V600E mutation in fine-needle aspirations of thyroid nodules. Chinese Journal of Ultrasonography 2015;(8):671-4. 